\title{
Welcome to the City: What Can Urban Centres Learn From Rural/Remote Experiences with Telepsychiatry?
}

Chetana Kulkarni, MD, Omar Ayad, M.Sc., Antonio Pignatiello, MD.

The Hospital for Sick Children, Toronto, Canada.

\section{Background:}

- Telepsychiatry enables an evidence-based service provision proven comparable to in-person models.

- For nearly 20 years, our TeleLink Mental Health Program has been successfully delivering user friendly, cost effective, sustainable and integrated child and youth mental health specialists services to rural and remote areas of Ontario, Canada.

- The program connects a specialist from a reputable urban paediatric health care institution ( 3 academic institutions) with community-based primary and mental health care workers and their patients in rural and remote areas of Ontario to provide direct/indirect clinical consults, re-occurring program consultations, and/or educational sessions on demand using video-conferencing.

\section{Why Urban Telepsychiatry?}

- Urban settings were initially excluded in service provision.

- However, in reality, wait times for in-person services to the same specialists for clients living in Toronto, remained at 6-12 months, and many children and youth do not receive the care they need.

- Many families thus present to emergency departments skipping local community resources.

- As a solution to access issues and long wait times, our TeleLink program implemented a 3 year project to provide services to clients in Toronto in 2017.

\section{Methods}

- Demographic data for Year 1 and 2 were collected

- Based on the Consolidated Framework for Implementation Research, we conducted semi-structured qualitative interviews. Sample included frontline referrers $n=\mathbf{1 0}$, CEO/Executive Directors of referring agencies $\mathbf{n}=\mathbf{9}$.

- Interviews aimed to uncover challenges, strengths and areas of improvement in the implementation.

- Thematic analysis of interview content.

- A full evaluation is currently under development in year 3 .

\section{$\underline{\text { Results }}$}

\begin{tabular}{|c|c|c|}
\hline \multirow{2}{*}{ Service Type } & \multicolumn{2}{|c|}{ Actual / Target } \\
\hline Clinical & Year 1 & Year 2 \\
\hline Program Consult & $7 / 100$ & $147 / 200$ \\
\hline Education & $1 / 4$ & $16 / 20$ \\
\hline
\end{tabular}

Actual Appointments vs Targets

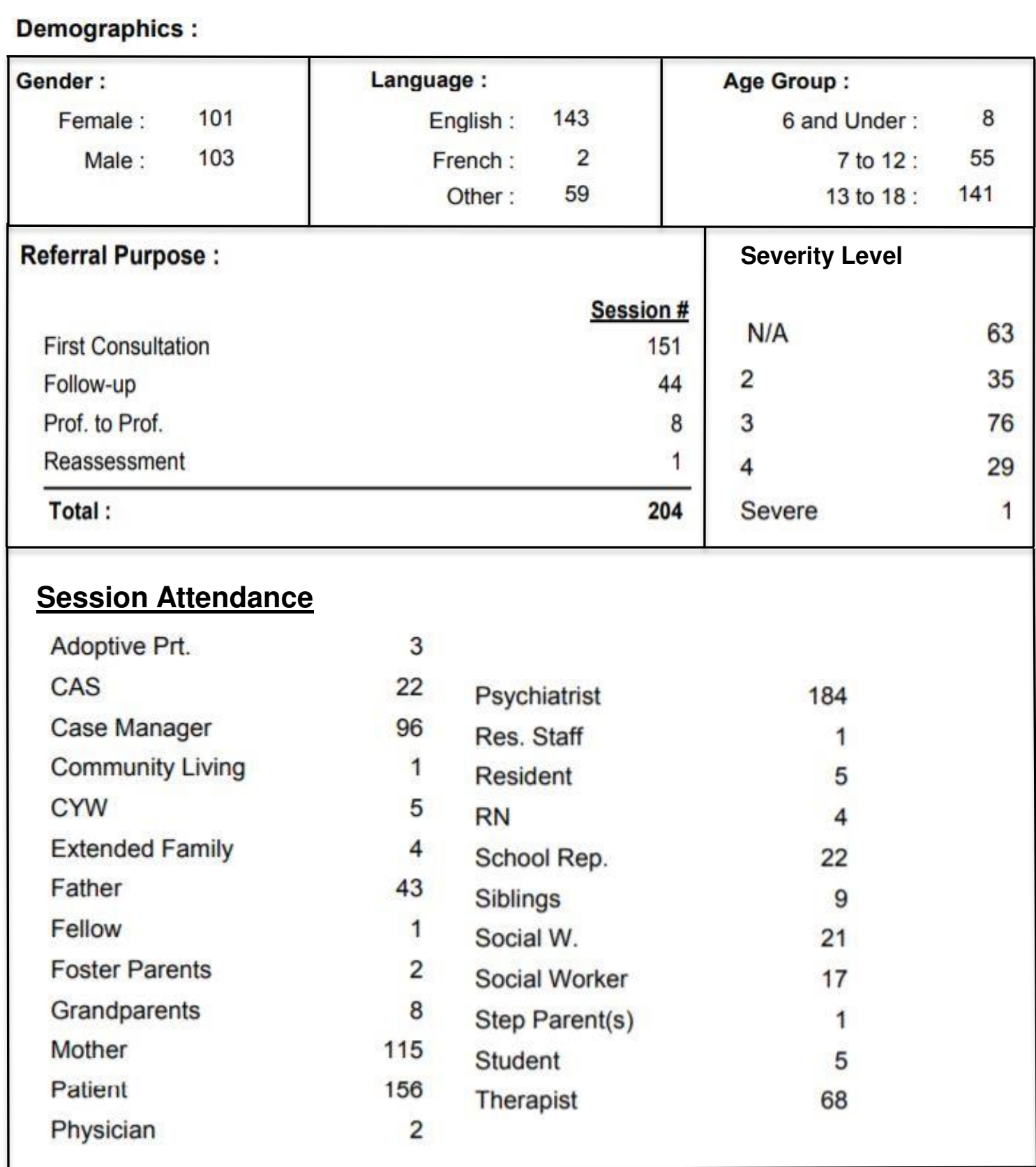

Monthly Clinical Appointment Trend

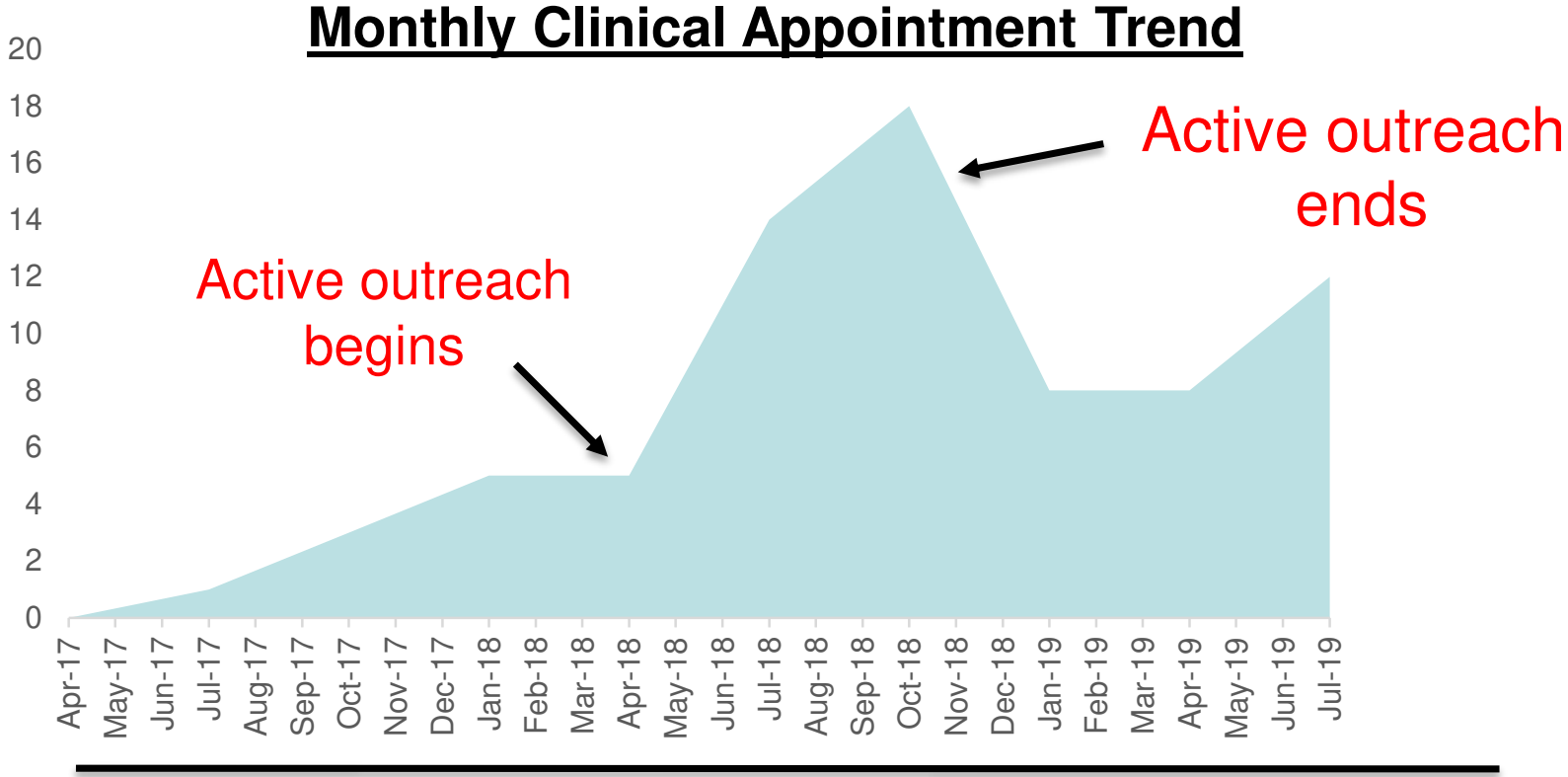

\section{Qualitative Analysis}

\section{Challenges}

Need for constant outreach and education.

- Requires Practice Shift in Toronto.

- Stigma.

- Family concerns with privacy and security.

- Needs relationship building for trust.

- High-turnover rates at agencies, requiring repeated outreach

- Designated remote sites initially presented a challenge. Services were offered virtually anywhere later in the implementation.

- Needs strong leadership support and championing.

\section{Conclusions}

We conclude that telepsychiatry represents a viable solution to addressing equitable access issues to psychiatric care in urban settings. However, simply building it does not guarantee referrals. Active outreach, championing and community education are critical enablers of success. 